\title{
Meron Pseudospin Solutions in Quantum Hall Systems
}

\author{
Sankalpa Ghosh and R. Rajaramant \\ School of Physical Sciences \\ Jawaharlal Nehru University \\ New Delhi 110067, INDIA
}

(September 17, 2018)

\begin{abstract}
In this paper we report calculations of some pseudospin textures for bilayer quantum hall systems with filling factor $\nu=1$. The textures we study are isolated single meron solutions. Meron solutions have already been studied at great length by others by minimising the microcopic Hamiltonian between microscopic trial wavefunctions. Our approach is somewhat different. We calculate them by numerically solving the nonlinear integro -differential equations arising from extremisation of the effective action for pseudospin textures. Our results can be viewed as augmenting earlier results and providing a basis for comparison.Our differential equation approach also allows us to dilineate the impact of different physical effects like the pseudospin stiffness and the capacitance energy on the meron solution.
\end{abstract}

\section{INTRODUCTION}

Recent years have witnessed the demonstration of topologically nontrivial spin textures like Skyrmions as physically important excitations in quantum Hall systems. It has been argued in a body of very interesting papers that such Skyrmions are not mere exotic theoretical curiosities but will actually be the lowest energy excitations in some situations ( such 
as for example filling factor $\nu=1$ and small Zeeman coupling) [1]. Furthermore, experimental evidence indicating the presence of such Skyrmionic excitations has also subsequently emerged [2]. Skyrmions of topological charge more than unity have also been studied [3].

These results were basically for mono-layers of electron gas in strong magnetic fields, but with spin degrees of freedom of the electrons treated dynamically. Meanwhile, the remarkable discoveries of the quantum Hall effect, originally found in single two dimensional electron layers, have also been extended to bilayer systems, thanks to the development of techniques for growing GaAs heterostructures containing two separated layers of twodimensional electron gas (see for example references [4]) . On the theoretical front, a large body of work has already been done on bilayer systems. An extensive list of references to this literature has been given in the lucid review of this subject by Girvin and MacDonald [5] and in the paper by Moon et al [6].

To simplify matters, one may begin analysing bilayer systems by assuming, to start with, that the electrons are spinless, i.e. that the spins of the electrons are fully polarised by the strong magnetic field and frozen as degrees of freedom. The idea would be to later incorporate the complications brought about by spin degrees of freedom after the spinless problem is understood. However, even when real physical spin is suppressed, it is helpful to view electrons in a bilayer system as carrying a "pseudospin" in addition to their spatial coordinates on the plane. The notion of such a pseudospin arises from mapping the bilayer spinless problem into a monolayer problem with spin [7]. In this mapping the layer degree of freedom is specified by a two-component pseudospinor whose up and down components refer to the amplitude for the electron to be in the first and second layers respectively. Such a mapping allows one to borrow for bilayer systems, the rich body of insights and results available from single layer systems with real spin. For instance, just as in case of electrons with real spin the combination of the Pauli principle and Coulomb interactions can lead to a ferromagnetic ground state, one can similarly expect the pseudospin of bilinear systems to also be fully "polarised" under corresponding circumstances. Such psuedospin magnetisation amounts to spontaneous phase coherence between the two layers, with many 
interesting consequences, discussed in the literature [5], [6].

Going beyond the ground state, the relevance of Skyrmions to systems with real spin has in turn prompted studies of similar topological excitations in spinless bilayer systems, but now involving pseudospin (See references [5], [6] and references given therein). Because of interplane-intraplane anisotropy in pseudospin stiffness in bilayer systems, as well the capacitance energy of carrying unequal charge density in the two layers, the effective Action governing pseudospin enjoys only $\mathrm{U}(1)$ symmetry of rotations about the z-axis (the direction perpendicular to the $\mathrm{x}-\mathrm{y}$ plane of the layers). Finiteness of energy requires that asymptotically the pseudospin must lie on the easy (x-y) plane. The basic topological excitations in that case are the so-called merons which are vortices in pseudospin with a winding number of one-half, and meron anti-meron bound pairs. Such a pair is topologically equivalent to Skyrmions and carries unit winding number. (For an introduction to such topological excitations, their winding numbers, requirements due to finite energy, etc. see reference [9].)

In this paper we obtain single meron solutions in bilayer systems with filling fraction $\nu=1$, for a range of different values of system parameters (such as the inter layer separation d) by numerically solving the integro differential equation obeyed by them. Even though a single meron by itself has a logarithmically divergent energy in the infinite volume limit it is nevertheless an object of interest. Physical meron anti-meron solutions which do have finite energy, are often treated as a bound pair of two extended interacting objects, namely the individual merons each with a "size" and a meron core energy . Merons are not rigid bodies but rather solutions of nonlinear wave equations; the notion of giving each of them a fixed size, shape and energy, although only approximate when they appear as a part of a larger solution containing other merons, is nevertheless useful in such Soliton physics. Single meron solutions allow us to obtain their size, shape and individual energy in an unambiguous way.

Single meron and bi-meron solutions have already been extensively studied in a body of papers by Girvin, MacDonald and co-workers [8] [10] and [6] . These calculations are based on optimising microscopic wavefunctions with respect to the microscopic interaction Hamiltonian. The emphasis is on determining the optimised energy of bimeron configurations and 
extracting individual meron core sizes and energies from them. Our work here relies heavily on the advances already made in these papers, and is to be viewed as something which will hopefully augment their calculations. We will obtain explicit meron solutions through a somewhat different route, by numerically integrating the nonlinear differential equations derived from the effective action obeyed by the pseudospin magnetic moments. Meron pro-

files are of some interest in their own right. [It is for instance not widely realised that unlike Skyrmions which exist even in the pure nonlinear sigma model (NLSM) limit, that model will not permit exact meron solutions. Meron solutions require departure from the NLSM model, which is provided by the capacitance energy and other anisotropies. We will explain this point further later on.] We will obtain our meron profiles for a range of values of the layer distance and study the way in which the solution is affected by the addition of leading corrections to the NLSM effective action. We also compare our results for the meron size and core energy with those obtained from other methods.

\section{RECAPITULATION OF THEORETICAL PRELIMINARIES}

The integro-differential equation which we will integrate to get our meron solutions is obtained by extremising an effective action which has already been derived by Moon et al [6] starting from the basic microscopic physics. See also Ezawa [11]. For completeness, and to set up the notation let us quickly recall their result. The pseudospin texture of a state is described by a classical unit vector $\vec{m}(\vec{r})$ which gives the local direction of the pseudospin. Here $\vec{r}$ is the coordinate on the x-y plane carrying the layers, while the magnetic field $\mathrm{B}$ is along the z-direction. The fully polarised "ferromagnetic" ground state $\left|\Psi_{0}\right\rangle$ corresponds to $\vec{m}$ pointing everywhere in the same direction, say, along the x-axis. Using this as the reference state, any other state with some arbitrary texture $\vec{m}(\vec{r})$ is given by performing a local pseudospin rotation on this uniform ground state :

$$
|\vec{m}(\vec{r})\rangle \equiv e^{-i \mathcal{O}}\left|\Psi_{0}\right\rangle
$$

Here the pseudospin rotation operator is, for infinitesimal rotations, 


$$
\mathcal{O}=\int d^{2} x(\hat{x} \times \vec{m}(\vec{r})) \cdot \vec{S}(\vec{r})
$$

where $\vec{S}$ is the local pseudospin operator given by $\vec{S}=\frac{1}{2} \psi_{\alpha}^{\dagger} \sigma_{\alpha \beta} \psi_{\beta}$ and $\psi_{\alpha}$ is the second quantised electron field operator with $\alpha$ denoting the pseudospin (layer) index. The effective action $I[\vec{m}]$ for any given pseudospin texture $\vec{m}(\vec{r})$ has already been derived by Moon et al [6] starting from the basic microscopic physics. See also Ezawa [11]. The microscopic Hamiltonian is

$$
H=H_{K}+H_{\text {int }}
$$

where

$$
H_{K}=\frac{1}{2 m} \sum_{\alpha=1}^{2} \int d^{2} x \psi_{\alpha}^{\dagger} D^{2} \psi_{\alpha}
$$

and,

$$
H_{\text {int }}=\frac{1}{2} \int d^{2} x d^{2} x^{\prime} \delta \rho_{\alpha}(\mathbf{x}) V_{\alpha \beta}\left(\mathbf{x}-\mathbf{x}^{\prime}\right) \delta \rho_{\beta}\left(\mathbf{x}^{\prime}\right)
$$

and $\delta \rho_{\alpha}$ is the local density operator minus the mean density in the $\alpha$ th layer. The interaction potential written here as a $2 \times 2$ matrix in pseudospin(layer) space is due to Coulomb forces between electrons in the same or different layers and is given by

$$
V_{\alpha \beta}\left(\mathbf{x}-\mathbf{x}^{\prime}\right)=\frac{\mathbf{e}^{2}}{\epsilon \sqrt{\left(\mathbf{x}-\mathbf{x}^{\prime}\right)^{2}+\mathbf{d}^{2}\left(1-\delta_{\alpha \beta}\right)}}
$$

where dis the layer separation.

The effective action $I[\vec{m}]$ is just the expectation value of the microscopic Hamiltonian in the state $|\vec{m}(\vec{r})\rangle$, but with all operators projected on to the lowest landau level (LLL). The detailed calculation is given in ref [6] and leads to a nonlocal action functional of $\vec{m}(\vec{r})$. Upon doing a gradient expansion, valid for slowly varying (long wavelength) textures, the leading terms are

$$
I(\vec{m})=\int d^{2} r\left[\frac{1}{2} \rho_{A}\left(\nabla m_{z}\right)^{2}+\frac{1}{2} \rho_{E}\left(\left(\nabla m_{x}\right)^{2}+\left(\nabla m_{y}\right)^{2}\right)+\beta m_{z}^{2}\right]+C[\mathbf{m}]
$$

where 


$$
C[\mathbf{m}] \equiv \frac{e^{2} d^{2}}{32 \pi^{2} \epsilon} \int d^{2} r \int d^{2} r^{\prime}\left(\frac{m_{z}(\mathbf{r}) \nabla^{2} m_{z}\left(\mathbf{r}^{\prime}\right)}{\mid\left(\mathbf{r}-\mathbf{r}^{\prime} \mid\right.}\right)
$$

and terms of order $\nabla^{3}$ and higher have been dropped. The constants appearing in this effective action are given in terms of microscopic interaction parameters. The constants $\rho_{A}$ and $\rho_{E}$ are pseudospin stiffness parameters whose physical origin is the exculsion principle (Hund's rule) mentioned earlier. They are given by

$$
\begin{aligned}
& \rho_{A}=\left(\frac{\nu}{32 \pi^{2}}\right) \int_{0}^{\infty} d k k^{3} V_{k}^{A} \exp \left(\frac{-k^{2}}{2}\right) \\
& \rho_{E}=\left(\frac{\nu}{32 \pi^{2}}\right) \int_{0}^{\infty} d k k^{3} V_{k}^{E} \exp \left(\frac{-k^{2}}{2}\right)
\end{aligned}
$$

where $V_{k}^{A}=2 \pi e^{2} /(\epsilon k)$ and $V_{k}^{E}=\exp (-k d)\left(2 \pi e^{2} / \epsilon k\right)$ are the Fourier transforms of the Coulomb interactions between electrons in the same and different layers respectively. All distances (and inverse wave vectors) are in units of the magnetic length $l$. The $\beta m_{z}^{2}$ term represents the so-called capacitance or charging energy needed to maintain unequal amounts of charge density in the two layers. Recall that the z-component of pseudospin represents the difference between the densities in the two layers. The constant $\beta$ is given by

$$
\beta=\left(\frac{\nu}{8 \pi^{2}}\right) \int_{0}^{\infty} d k k\left(V^{z}(0)-V^{z}(k)\right) \exp \left(\frac{-k^{2}}{2}\right)
$$

where $V_{k}^{z}=\frac{1}{2}\left(V_{k}^{A}-V_{k}^{E}\right)$.

\section{THE FIELD EQUATION AND ITS SOLUTIONS}

The nonlinear partial differential equations that have to be obeyed by all slowly varying spin textures, whether they be single merons or multi merons is obtained by extremising this

effective action $I[\vec{m}]$ with respect to $\vec{m}(\vec{r})$. In doing this extremisation one has to respect the condition that $\vec{m}$ is a unit vector. We handle this by writing $\vec{m}$ in terms of independent fields $m_{z}$ and $\alpha$ such that

$$
\mathbf{m}=\left(\sqrt{1-m_{z}^{2}} \cos \alpha, \quad \sqrt{1-m_{z}^{2}} \sin \alpha, m_{z}\right)
$$


As one can see $\alpha=\tan ^{-1}\left(\frac{m_{y}}{m_{x}}\right)$ is the azimuthal angle of the vector $\vec{m}$ in the x-y plane. Upon rewriting $I[\vec{m}]$ in terms of the independent fields $m_{z}(\vec{r})$ and $\alpha(\vec{r})$, and minimising with respect to them we get the field equations

$2 \beta m_{z}-\rho_{A} \nabla^{2} m_{z}-\rho_{E} m_{z}\left(\frac{\left(\nabla m_{z}\right)^{2}}{\left(1-m_{z}^{2}\right)^{2}}+\frac{m_{z} \nabla^{2} m_{z}}{1-m_{z}^{2}}+\nabla^{2} \alpha\right)+\frac{e^{2} d^{2}}{16 \pi^{2} \epsilon} \int d^{2} r \int d^{2} r^{\prime}\left(\frac{\nabla^{2} m_{z}\left(\mathbf{r}^{\prime}\right)}{\left|\mathbf{r}-\mathbf{r}^{\prime}\right|}\right)=0$

and

$$
\vec{\nabla} \cdot\left[\left(1-m_{z}^{2}\right) \vec{\nabla} \alpha\right]=0
$$

We will numerically solve these coupled equations for the variables $m_{z}$ and $\alpha$. In the case of the Skyrmion and bi-meron solutions people have found it helpful to begin with the Isotropic Nonlinear Sigma model (NLSM) limit of the effective action and field equations, whose analytical solutions are exactly known. These exact solutions offer a starting point for setting up trial functions for numerically solving the full equations. In the case of the meron however, there is no exact single meron solution to the NLSM. To see this let us briefly consider the NLSM limit.

\section{A. Absence of single-merons in the NLSM limit}

The SU(2) invariant NLSM limit is obtained when the layer separation vanishes $(d / l \rightarrow$ $0)$. At the level of the microcopic Hamiltonian one can see that it is rotationally $(\mathrm{SU}(2))$ invariant in pseudospin in the limit of zero separation. The potential energy in (2.5) and (2.6) is layer independent when $d=0$, and in the LLL approximation the kinetic term (2.4) involving the magnetic field is just a constant . This symmetry is also reflected in the effective action $I[\vec{m}]$. With $d=0$, we see from their definitions that $\rho_{A}=\rho_{E}$ while $\beta=0=C[\vec{m}]$. Then the effective action in (2.7) reduces to just the NLSM. The equation for $m_{z}$ in turn reduces to

$$
\frac{m_{z}\left(\nabla m_{z}\right)^{2}}{\left(1-m_{z}^{2}\right)^{2}}+\frac{\nabla^{2} m_{z}}{1-m_{z}^{2}}+m_{z} \nabla^{2} \alpha=0
$$


while 3.3 remains the same. The NLSM has been fully solved [9] and its solutions are most conveniently described in terms of the complex field w(z) defined by

$$
w(z) \equiv \frac{m_{x}+i m_{y}}{\left(1-m_{z}\right)}
$$

where $\mathrm{z}=\mathrm{x}+\mathrm{iy}$. Any analytic function $\mathrm{w}(\mathrm{z})$ will be a solution of the NLSM. In particular, the single Skyrmion solution is $w(z)=\frac{z}{\lambda}$, which corresponds in polar coordinates $(r, \theta)$ to the circularly symmetrical function with

$$
m_{z}=\frac{r^{2}-\lambda^{2}}{r^{2}+\lambda^{2}} \quad \text { and } \quad \alpha=\theta
$$

It can be checked that (3.6) satisfies equation (3.4)

Now, isolated meron or anti-meron solutions may be defined by the boundary conditions

$$
\begin{gathered}
m_{z}(r=0)= \pm 1 \\
\text { and } m_{z}=0 ; m_{x}+i m_{y}=\exp ( \pm i \theta), \quad \text { as } r \rightarrow \infty
\end{gathered}
$$

Solutions with such boundary conditions may or may not exist for a given equation. In fact we will argue that the basic NLSM has no meron solutions. But the NLSM does have a Skyrmion solution of the form (3.6). The notion of a meron stems from the portion of the Skyrmion function (3.6) from $\mathrm{r}=0$ to $r=\lambda$. In this portion the circle of radius $\lambda$ in the $\mathrm{z}$ plane is mapped onto the unit circle in the w-plane. The spin begins at $\mathrm{r}=0$ with $m_{z}=-1$ starts tilting towards the $\mathrm{x}-\mathrm{y}$ plane, and by $r=\lambda$ is lying entirely on the $\mathrm{x}-\mathrm{y}$ plane, pointing radially outward. The topological number density when integrated over this portion, is onehalf. Sometimes this portion is called a meron, i.e. half a Skyrmion. However, it must be emphasized that this object corresponds only to a piece of the full Skyrmion function and is not a solution to the field equations in the full $x-y$ plane. In contrast an "isolated single meron solution" to the field equation, while qualitatively similar, should span the whole x-y plane and not just a portion of it. That is, it should satisfy the boundary condition (3.7) at infinity and not at some finite $r=\lambda$. Such a solution does not exist. The easiest way to understand this is to note that there exists no analytical function $\mathrm{w}(\mathrm{z})$ which maps the 
z-plane into a unit circle in the w-plane. Equivalently, if one tried to solve the differential equation 3.4 starting with $m_{z}=-1$ at $\mathrm{r}=0$ and any positive slope, one will always end up with a Skyrmion, i.e. with $m_{z}=+1$ at $r=\infty$. Note that this is not directly related to the fact that an isolated meron ,if it existed, would have a logarithmically divergent energy. Solutions can exist to diferential equations with some boundary conditions, even if they have infinite value for the energy functional. But in the case of merons, no such solution exists for the NLSM.

\section{B. Meron solutions when $d \neq 0$}

When the layer separation $d$ is non-zero, then the full effective action (2.7) comes into play and we have no longer just the NLSM. The arguments of the preceding subsection do not hold and now meron solutions can exist. See Affleck [12 for arguments show that in fact they will exist when $\beta$ in eq (3.2) is non-zero.

We will look for spherically symmetric solutions with $\alpha(r, \theta)=\theta$ which will satisfy

eq(3.3) provided $m_{z}$ depends only on the radius $\mathrm{r}$. Then eq (3.2) requires that $m_{z}(r)$ satisfy 


$$
\begin{array}{r}
2 \beta m_{z}-\rho_{A}\left(\partial_{r}^{2} m_{z}+\frac{1}{r} \partial_{r} m_{z}\right)-\rho_{E}\left(\frac{m_{z}\left(\partial_{r} m_{z}\right)^{2}}{\left(1-m_{z}^{2}\right)^{2}}\right. \\
\left.+\frac{m_{z}^{2}\left(\partial_{r}^{2} m_{z}+\frac{1}{r} \partial_{r} m_{z}\right)}{1-m_{z}^{2}}+\frac{m_{z}}{r^{2}}\right) \\
+\frac{e^{2} d^{2}}{16 \pi^{2} \epsilon} \int d^{2} r^{\prime}\left(\frac{\nabla^{2} m_{z}\left(\vec{r}^{\prime}\right)}{\left|\vec{r}-\vec{r}^{\prime}\right|}\right)=0
\end{array}
$$

The parameters $\rho_{A}, \rho_{E}$ and $\beta$ in the equation are calculated for each value of the layer separation $d$ from their definitions (2.9 and 2.10).

As it stands (3.8) is a nonlinear integro-differential equation. It is the nonlocal integral in the last term which represents the most difficult part of this equation. To start with let us neglect this term. We are then left with a nonlinear ordinary second order differential equation given by

$$
2 \beta m_{z}-\rho_{A}\left(\partial_{r}^{2} m_{z}+\frac{1}{r} \partial_{r} m_{z}\right)-\rho_{E}\left(\frac{m_{z}\left(\partial_{r} m_{z}\right)^{2}}{\left(1-m_{z}^{2}\right)^{2}}+\frac{m_{z}^{2}\left(\partial_{r}^{2} m_{z}+\frac{1}{r} \partial_{r} m_{z}\right)}{1-m_{z}^{2}}+\frac{m_{z}}{r^{2}}\right)
$$

We will first solve this equation and then later on return to the full equation (3.8) including the last integral term. To solve (3.9) numerically we merely start with a boundary value and slope for $m_{z}$ at $\mathrm{r}=0$, obtain $\partial_{r}^{2} m_{z}$ using the equation (3.8), use this to get the value and slope at the next point in $\mathrm{r}$, and thus proceed towards $r \rightarrow \infty$. One can see that the aymptotic behaviour of $m_{z}(r)$ must be

$$
\begin{aligned}
m_{z} & =1-a r^{2}+O\left(r^{3}\right) \quad \text { as } \quad r \rightarrow 0 \\
\text { and } \quad m_{z} & \sim \exp (-k r) \quad \text { as } \quad r \rightarrow \infty
\end{aligned}
$$

where $k \equiv\left(\sqrt{\frac{2 \beta}{\rho_{A}}}\right)$ and $a$ is a constant for a given layer separation $\mathrm{d}$. The absence of an $O(r)$ term in $m_{z}(r \simeq 0)$ follows from the requirement that our solution not have a cusp at $\mathrm{r}=0$. One may be concerned that the last term (proportional to $\rho_{E}$ ) in eq (3.9) appears to have pieces with an $r^{-2}$ singularity at $\mathrm{r}=0$. It must be remembered that $\left(1-m^{2}\right)^{-1}$ also behaves as $r^{-2}$. However it can be checked that the requirement (3.10) ensures that the $r^{-2}$ singularities in the different pieces cancel one another, regardless of the value of the constant $a$. This constant $a$ is adjusted so that $m_{z}$ exponentially falls to zero as $r \rightarrow \infty$ as required. 


\section{RESULTS AND DISCUSSION}

The resulting solutions of eq (3.9) for $m_{z}(r)$ are plotted in figure 1 for a range of 6 different values of $d=0.24,0.50,0.625,0.78,1.00,1.20$ and 1.50 respectively in units of the magnetic length $l$. Each solution falls monotonically to zero as $r$ increases with an exponential fall as $r \rightarrow \infty$ as required by $(3.10)$. Comparing solutions for different values of the interlayer distance $d$, we see that the width (mean radius) of the solution monotonically decreases with $d$, the outermost curve shown in fig. 1 corresponding to $d=0.24$ and the inner most to $d=1.50$. The physics behind this is the minimisation of the capacitance energy $\beta m_{z}^{2}$, which would tend to bring $m_{z}$ down to zero as quickly as possible, in contrast to the gradient (stiffness) terms would tend to reduce $m_{z}$ as slowly as possible from its starting value of unity at $\mathrm{r}=0$. As $d$ increases so does $\beta$, while $\rho_{E}$ falls with $d$ and $\rho_{A}$ is independent of $d$ (see equations (2.10) and 2.10). Therefore the relative importance of the capacitance energy to the stiffness terms increases with $d$, and hence the solutions fall to zero faster.

In fig. 2 we plot the meron core radius $R_{m c}$, which we define following the literature [10] as the radius at which $m_{z}$ equals 0.1 . As noted above this radius falls monotonically with d. In fig.2 we show our values of $R_{m c}$ for 8 different values of $d$, while for comparison the results of Yang and MacDonald (extracted from fig.2 of ref. [10]) are shown in the form of a curve. They consider their results to be reliable in the region $0.5 \leq \mathrm{d} \leq 1.2$. In this range our values of $R_{m c}$ are of the same order of magnitude as theirs but about 30 to 40 percent smaller . The qualitative behavior as a function of $d$ is very similar.

The energy of the meron, i.e. the value of the effective action for the meron solution will diverge logarithmically if evaluated over the entire plane because of its nonvanishing azimuthal gradient. However the notion of a "meron core energy" applies to the energy of the meron function evaluated upto $r=R_{m c}$. In fig.3 we plot $E_{m c}$ as a function of $d$.

Since single merons have, strictly speaking, divergent energy, physical finite energy excitations involving merons must come in the form of meron-antimeron pairs, which do have finite energy . Using $E_{m c}$ one can make a phenomenological estimate of the energy 
$E_{M P}$ of such meron pairs as pointed out in references [6] and [10]. Following them we can approximately write the energy of a pair separated by a distance $\mathrm{R}$ in the form

$$
E_{M P}=2 E_{m c}+2 \pi \rho_{E} \ln \left(R / R_{m c}\right)+\frac{e^{2}}{4 \epsilon R}
$$

The optimal value $R^{*}$ of the meron separation can be obtained by minimising this energy with respect to $\mathrm{R}$ to get $R^{*}=\frac{e^{2}}{\left(8 \pi \epsilon \rho_{E}\right)}$. Substituting this value of $R^{*}$ into the above equation we get

$$
E_{M P}=2 E_{m c}+2 \pi \rho_{E}\left(1+\ln \left(\frac{e^{2}}{8 \epsilon \rho_{E} R_{m c}}\right)\right)
$$

Table 1 displays the values of meron core radius, core energy, the merom pair separation and energy as obtained by us, for different values of $d$. These may be compared to values obtained by others using microscopic Hartree Fock methods. We see that for the lower values od $d$, such as 0.24 and 0.5 , the meron separation $R^{*}$ is either smaller or of the same order as the core radius $R_{m c}$. The approximation of two well separated merons is clearly not good at these values of $d$. Equations such as (4.1) should be used only at layer separation $d$ beyond 0.6. This is in agreement with the findings of Yang and MacDonald [10].

Finally we include the non-local integral (the last term) in the original field equation (3.8) which we had neglected so far. It arose in this equation as the functional derivative $\frac{\delta C[\vec{m}]}{\delta m_{z}}$ of the term $C[\vec{m}]$ in the Effective action (2.7). The presence of this term not only makes the equation more difficult to solve, but also alters to some extent the nature of the solution. Notice that this term behaves for large $r$ as $\frac{C}{r}$ where $C \propto \int d^{2} r^{\prime} \nabla^{2} m_{z}\left(\vec{r}^{\prime}\right)$. This $\frac{1}{r}$ behavior will not permit an exponential fall off of $m_{z}$ as had happened in the solutions in the absence of this term, shown in figure 1. Instead the solution can be expected to fall to zero asymptotically only as some inverse power of $r$. Further, the value of the constant $\mathrm{C}$ for most values of $d$ of interest is positive. For large $r$ the solution for $m_{z}$, may therefore approach zero from below.

These expectations are supported by the numerical solution of the integro-differential equation (3.8). We solve it by the following iterative procedure. Start with the solution $m_{z}^{0}$ 
of eq(3.9) , where the above-mentioned integral has been altogether neglected. Evaluate the integral by inserting $m_{z}^{0}$ in its integrand. Multiply the resulting integral by a prefactor $\alpha=$ 0.1 , add it to eq.(3.9), and solve the equation again to obtain $m_{z}^{\alpha}$. Solve the equation again after inserting this $m_{z}^{\alpha}$ into the integral, call the solution $m_{z}^{2 \alpha}$, and repeat this procedure ten times until you get $m_{z}^{10 \alpha}$. Since $10 \alpha=1$ this should yield a good approximation to the full equation (3.8). One could have used this iterative procedure in smaller steps, but we find the step size of $\alpha=0.1$ is small enough for the procedure to converge smoothly .

In figure 4 we compare the solution so obtained for the full equation (3.8) with the "unperturbed" solution (of the simpler eq (3.9) discussed earlier), for two values of $d$ equal to 0.625 and 1.20 . One can see that the full solution reaches zero, and then after turning negative, returns to zero asymptotically but more slowly than the unperturbed solution. In other words the pseudospin, which starts out pointing along the $\mathrm{z}$-axis at $\mathrm{r}=0$, gradually falls towards the $\mathrm{x}-\mathrm{y}$ plane, lies entirely on the $\mathrm{x}-\mathrm{y}$ plane at some finite radius, then starts bending towards the negative z-axis, then reverses its behavior to finally asymptotically come back to the $\mathrm{x}-\mathrm{y}$ plane at infinity. We see no objection to such a meron solution. But the notion of a core may be less meaningful now. One could formally evaluate the "meron core radius $R_{m} c$ " of this new solution defined once again as the radius at which $m_{z}=0.1$, and a corresponding core energy. But with the solution continuing to be substantially nonzero at radii quite a bit larger than such an $R_{m} c$, the concept of a core is less significant for such slowly falling solutions.

\section{Acknowledgement}

R.R acknowledges many valuable discussions with Dr Shivaji Sondhi on Hall systems. We also thank Dr Subir Sarkar for his unstinting help in doing the numerical work here. The work of S.G. is supported by a CSIR award no.9/263(225)/94-EMR-I .dt.2.9.1994. 
Table 1: Meron core radius $\left(R_{m c}\right)$, meron-antimron separation $\left(R^{*}\right)$,meron core energy $\left(E_{m c}\right.$ ) and meron pair energy $\left(E_{M P}\right)$ as a function of layer separation. The unit of energy is $\frac{e^{2}}{\epsilon l}$ and the unit of length is $l$

\begin{tabular}{|c|c|c|c|c|}
\hline $\mathrm{d}$ & $R_{m c}$ & $R^{*}$ & $E_{m c}$ & $E_{M P}$ \\
\hline .2400 & 5.07276 & 2.3133 & 0.145487 & 0.314187 \\
\hline .5000 & 3.07692 & 3.52110 & 0.105005 & 0.290580 \\
\hline .6250 & 2.70270 & 3.9868 & 0.101588 & 0.290250 \\
\hline .7810 & 1.99584 & 4.88985 & 0.084911 & 0.266760 \\
\hline 1.000 & 1.41372 & 6.42600 & 0.067163 & 0.232140 \\
\hline 1.200 & 1.14761 & 8.15756 & 0.057474 & 0.205750 \\
\hline 1.500 & 0.873181 & 11.3682 & 0.042215 & 0.163039 \\
\hline
\end{tabular}




\section{FIGURES}

FIG. 1. The solution $m_{z}(r)$ of equation(3.9). The six curves correspond to layer separation $d$ equal to $0.24,0.50,0.625,0.78,1.00,1.20$ and 1.50 respectively in units of the magnetic length l. The width of the curves decreases monotonically as as $d$ increases, with the outermost curve for $d=0.24$ and the innermost to $d=1.50$

FIG. 2. Meron core radius $R_{m c}$ for 8 different values of the layer separation $d$, all in units of l. The solid curve gives the results obtained in ref.10

FIG. 3. Meron core energy $E_{m c}$ as a function of layer separation

FIG. 4. $m_{z}(r)$ versus $r$. The continuous lines give the solution of eq(3.8) which includes the integral term and, for comparison, the dotted curves depict the solution of (3.9) which does not include this term. Figure (a) corresponds to $d=0.625$ and figure (b) to $d=1.2$ 


\section{REFERENCES}

* email: doug@jnuniv.ernet.in

[1] S.L.Sondhi, A.Karlhede, S.A.Kivelson and E.H. Rezayi, Phys.Rev. B 47, 16419, (1993); H.A.Fertig, L.Brey, R. Cote, and A.H. MacDonald, Phys.Rev. B 50, 11018, (1994). See also E.H.Rezayi, Phys. Rev. B 36, 5454, (1987) and D.-H. Lee and C.L.Kane Phys.Rev. Lett 64, 1313, (1990).

[2] S.E.Barrett, G.Dabbagn, L.N.Pfeiffer, K.W.West and R.Tycko, Phys.Rev.Lett.,74, 5112, (1995); A.Schmeller, J.P.Eisenstein, L.N.Pfeiffer, and K.W.West, Science, 268, 1460, (1995); E.H.Aifer, B.B.Goldberg and D.A.Broido, Phys. Rev. Lett., 76, 680, (1996).

[3] D.Lilliehook, K.Lejnell, A.Karlhede and S.L.Sondhi, "Quantum Hall Skyrmions with Higher Topological Charge", preprint cond-mat 9704121 (1997)

[4] J.P.Eisenstein, G.S.Boebinger, L.N.Pfeiffer, K.W.West and Song He, Phys.Rev.Lett.68, 1383, (1992) ; S.Q. Murphy, J.P.Eisenstein, G.S.Boebinger, L.N.Pfeiffer and K.W.West, Phys.Rev.Lett., 72, 728, (1994); Y.W.Suen, L.W.Engel, M.B.Santos, M.Shayegan and D.C.Tsui, Phys.Rev.Lett., 68, 1379, (1992); G.S.Boebinger H.W.Jiang, L.N.Pfeiffer and K.W.West, Phys.Rev.Lett., 64, 1793, (1990).

[5] S.M.Girvin and A.H.MacDonald, "Multi-Component Quantum Hall Systems : The Sum of Their Parts and More ", in Novel Quantum Liquids in Low-Dimensional Semiconductor Structures, S.D.Sarma and A.Piczuk Eds., Wiley (New York), 1995.

[6] K.Moon, H.Mori, Kun Yang, S.M.Girvin, A.H.MacDonald, L.Zheng D.Yashioka and Shou-Cheng Zhang, Phys.Rev. B 51, 5138 , (1995).

[7] A.H.MacDonald, P.M.Platzman and G.S. Boebinger Phys. Rev. Lett., 65, 775, (1990).

[8] L.Brey, H.A.Fertig, R.Cote and A.H.MacDonald, Phys. Rev. B. 54, 16888 , (1996). 
[9] R.Rajaraman, Solitons and Instantons, North Holland, Amsterdam, (1982).

[10] Kun Yang and A. H. MacDonald, Phys. Rev. B 51, 17247, (1995)

[11] Z.F.Ezawa, Phys.Rev. B 55, 7771, (1997).

[12] Ian Affleck, Phys. Rev. Lett.,56, 408, (1986). 


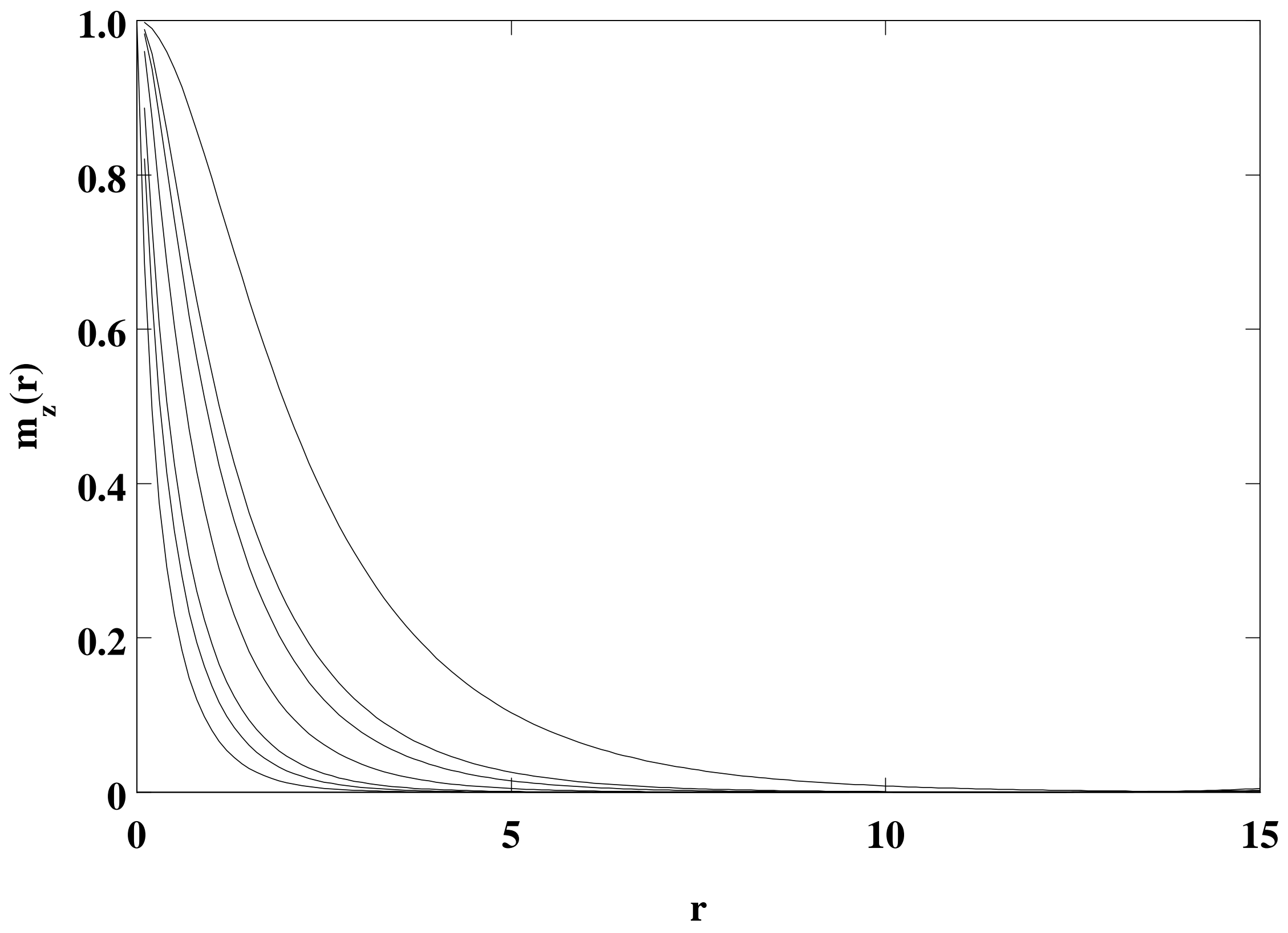




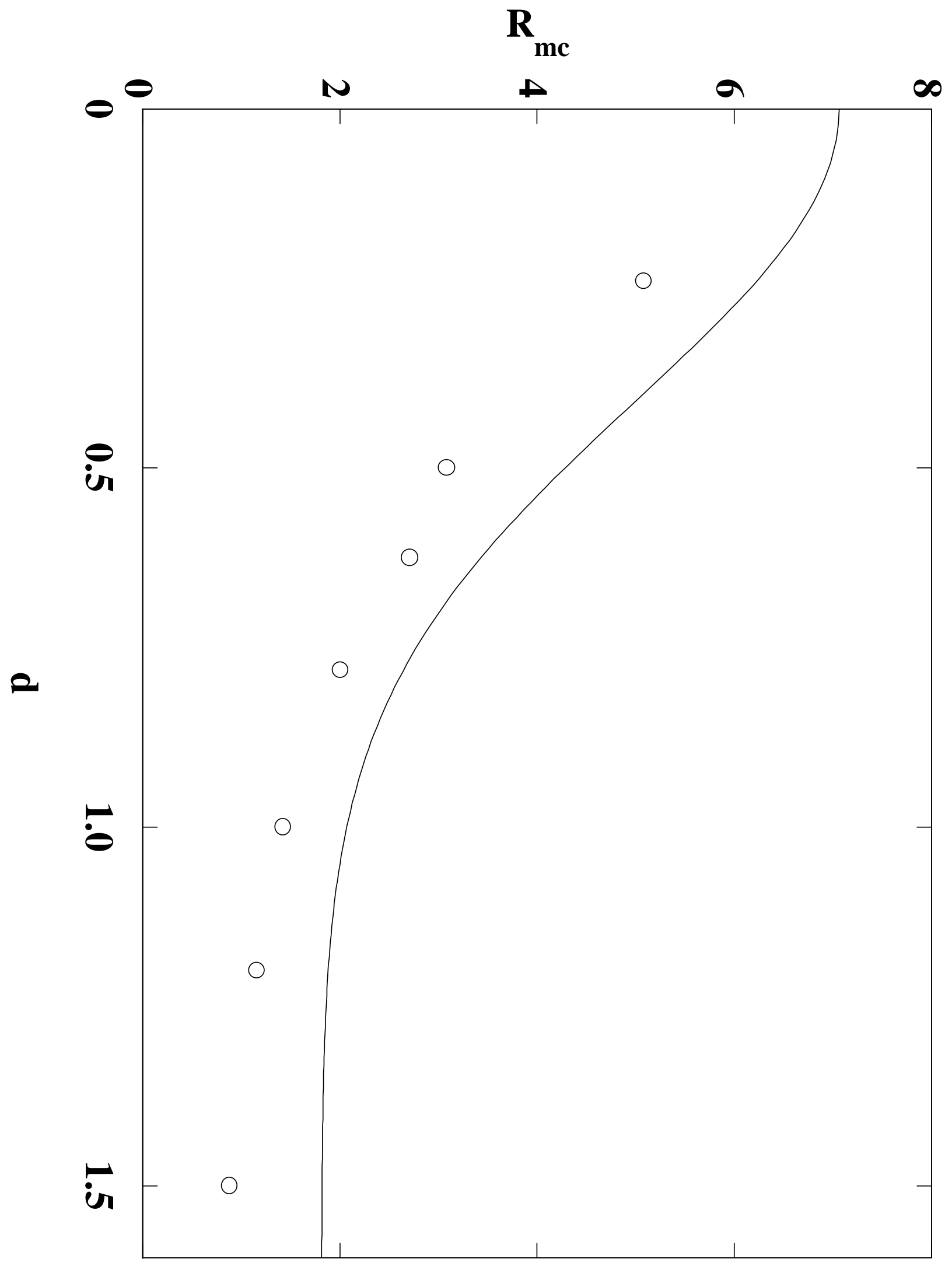




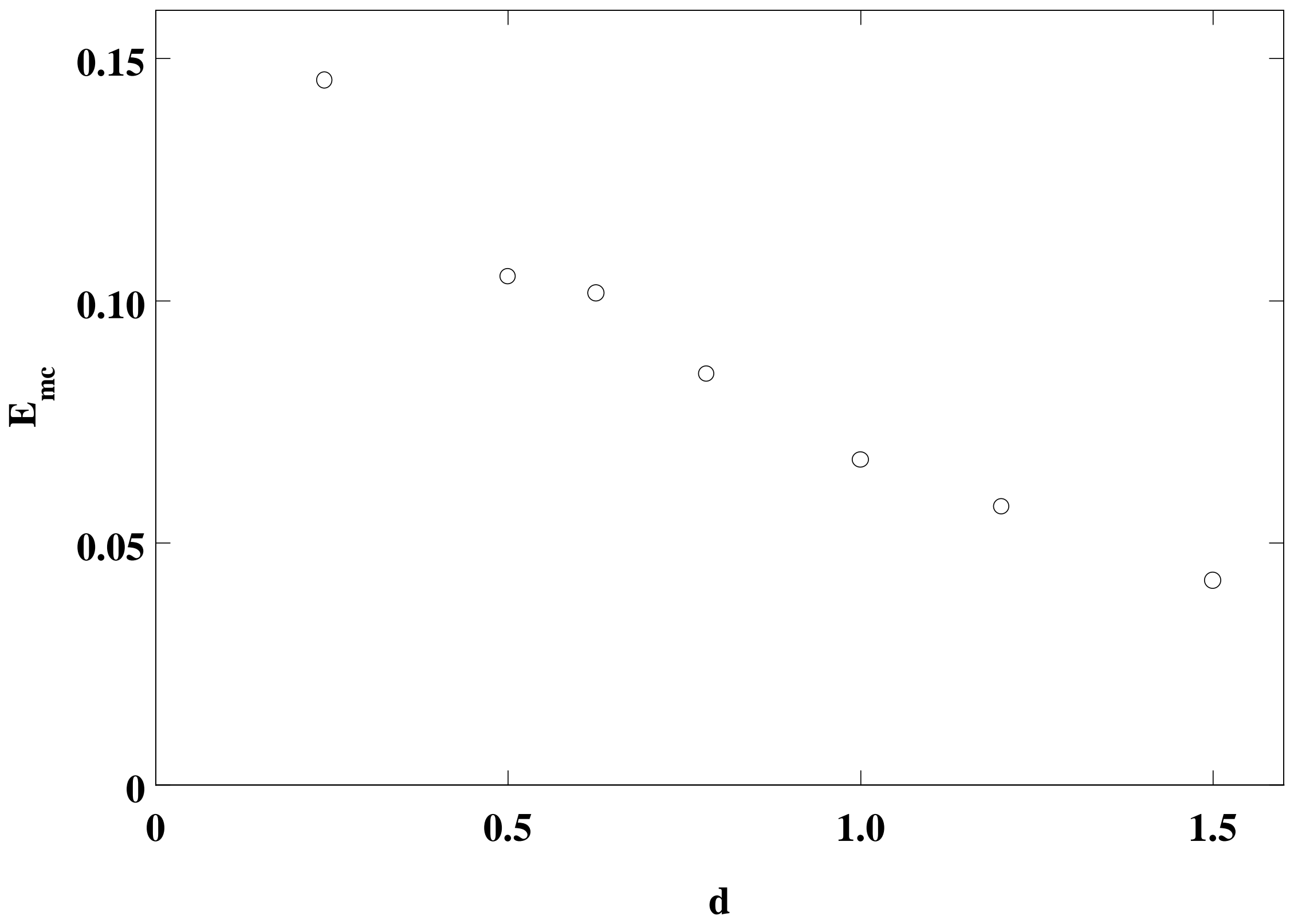



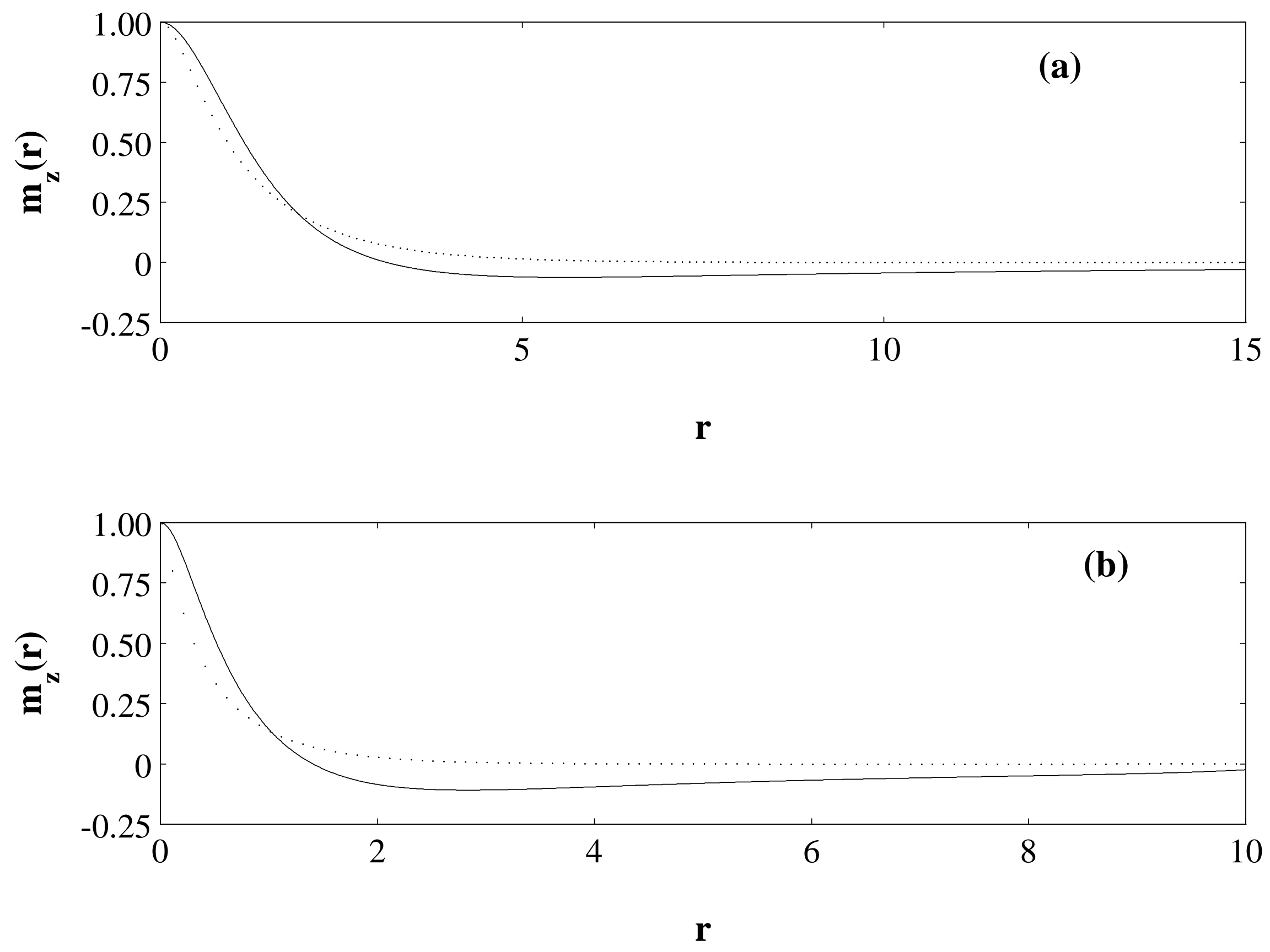